\title{
Automatic Plant Cultivation System (Automated Plant Factory)
}

\author{
Hitoshi OHARA ${ }^{1}$, Tatsuya Hirai ${ }^{1}$, Kouji KounO ${ }^{1}$ and Yoshifumi NishiURA ${ }^{2}$ \\ 'Tsubakimoto Chain Co., Agribusiness Department, 3-3-3 Nakanoshima, Kita-ku, Osaka 530-0005, Japan \\ ${ }^{2}$ Osaka Prefecture University, 1-1 Gakuen, Naka-ku, Sakai, Osaka 599-8531, Japan
}

(Received February 5, 2014; Accepted February 27, 2015)

\begin{abstract}
This paper describes a newly developed automated plant cultivation system consisting of a simple conveyor robot servicing a multi-shelf rack containing cultivation trays. The robot is situated between two multi-shelf racks, enabling easy access to any tray in any rack. Each multi-shelf rack is divided into sections and each section has artificial lighting at the top and nutrient solution circulated through its cultivation tray. This is a flexible system that has the ability to move plants between any sections in the rack by means of program commands that have been developed specifically for cultivation processes. The cultivation system described here has been operating in the Plant Factory Research Center of Osaka Prefecture University as a novel automated system of lettuce cultivation which requires no manual intervention during its 15 -day cultivation process, from seedling planting until harvesting.
\end{abstract}

Keywords : automatic transfer machine, flexible system, lettuce cultivation, multi-shelf rack, simple conveyor robot

\section{INTRODUCTION}

Plant factories have been arousing much interest as possible solutions to food crop cultivation problems, as there are issues entering the industry such as safety, security, supply stability, labor cost reduction, and the problem of less and less new farmers. Takatsuji et al. (1983) reported the international trend and problems in vegetable factory. They showed that the serious problem is the cost of both initial devices and running energy and materials. The control system in plant culture and speaking plant approach technologies were introduced as new processes in cultivation for plants by Hashimoto (1985). Ogura (2011) reported the history of plant factories in Japan and showed the works those are required the automation in plant factory or not. The Tsubakimoto Company has been developing an automated plant factory which meets the current demands of plant crop production, such as improved productivity, high yield rate, and stable product quality, based on our experience of large-scale plant cultivation, including plant and transportation/energy-saving systems in the 2000s.

Currently, many plant factories that use artificial light for indoor cultivation are gaining attention, and most of them are of the multi-shelf rack type. However, in the industries it is also well acknowledged that these systems are often highly labor-intensive, and the working conditions need improvement. These conditions are typically represented by repetitive work, with workers often in semicrouching positions, or reaching up to hazardous elevated areas. It is expected that automation will play an increasing role in future developments, along with the needs of higher productivity, scaling of cultivation, and improved working conditions.

The plant factory ultimately aims for systematic production based on sustainable plant cultivation. Automated factories should realize this goal, with consideration given to cost effectiveness, clean and safety, including requirements for simple, easy operation and bacteria-free.

We have recently developed a plant factory model (Fig. 1) which was exhibited by Osaka Prefecture University in Osaka Pavilion at Shanghai Expo in 2010. This system is reported by Nikkei technology News in 2010. This model is a compact automatic cultivation system combining hydroponics, an operation robot, and a transfer machine, demonstrating one of the new production methods for leaf vegetables to the expo visitors.

In 2011, we incorporated the technology of the Shanghai Expo model into a multi-story warehouse type of automated cultivation system and reported in the annual meeting of the Japanese Society of Agricultural Biological and Environmental Engineers and Scientists at Tokyo in 2012. This novel cultivation system utilizes artificial light, and automatic operation resulting in improved harvest volume per unit area. This system was delivered to the Plant Factory Research Center of Osaka Prefecture University.

\section{OVERVIEW OF THE MULTI-STORY WAREHOUSE TYPE SYSTEM}

This system is intended for cultivating leaf vegetables such as leaf lettuce, and is composed of a 15-stage multishelf rack for plant nursing and an automatic transfer unit to freely move the growing plants in the multi-shelf rack. The general process for growing vegetables starts from

Corresponding author: Hitoshi Ohara, fax: + 81-6-6441-0489, e-mail : hitoshi.ohara@gr.tsubakimoto.co.jp 
planting (seeding), and after growth to a certain height, proceeding through settled planting stages, until the plants are ready for harvest. The system has realized completely automatic cultivation where no manual labor is required from settled planting to harvest.

Figure 2 shows the configuration of the multi-story warehouse type of automatic cultivation system.

The automatic transfer unit, a core component of the system, moves plants freely in a 3-axis $x, y, z$, coordinate system, that is, backward/forward, right/left, and up/down. The controller mounted on the automatic transfer unit controls the movement of plants to/from and within the rack.

It is obvious that the plant factory requires lighting and air conditioning technologies. However, here we rather focus on the technology of transferring a plant to a target position.

After a plant grows to approximately $50 \mathrm{~mm}$ height in a separate nursery room, no less than 20 seedling locations are created in a plant cultivation panel (this is the settling

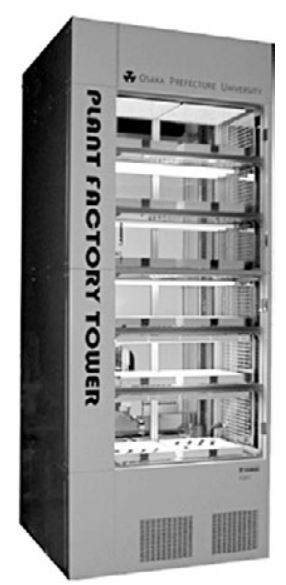

Fig. 1 Exhibition model of the Plant Factory at the Shanghai Expo. Dimensions: 1300 (W) $\times 1300$ (D) $\times 3200(\mathrm{H})$ (mm). stage). The purpose of the cultivation panel is to maintain the seedling posture and the spacing between each of the seedlings. The plants are then placed on the cultivation tray which holds the nutrient solution.

Once the cultivation trays containing the seedlings are placed at the inlet/outlet (Fig. 2), the trays are transferred to the top stage of the multi-shelf rack. Each day, each set (a stage) of trays is moved down by one level. All of these operations are performed by the automatic transfer unit. When this process is repeated over and over, and a stage of trays reaches the bottom, the plants in this stage can be harvested.

This system laterally combines a row of six cultivation trays on each side of the automatic transfer unit, giving a total of 12 trays for each stage and 15 stages vertically. The production capacity is 250 plants per day for leaf lettuce. The combined structure accommodates production scale simply by increasing or decreasing the number of rows of shelves.

Figure 3 shows the configuration of plants, the cultivation panel, cultivation tray, and below in Fig. 4, the transfer frame during transfer.

At transfer, each component uses one set integrally enclosed in a transfer frame. The NFT (Nutrient Film Technique) system is employed, in which the plant roots

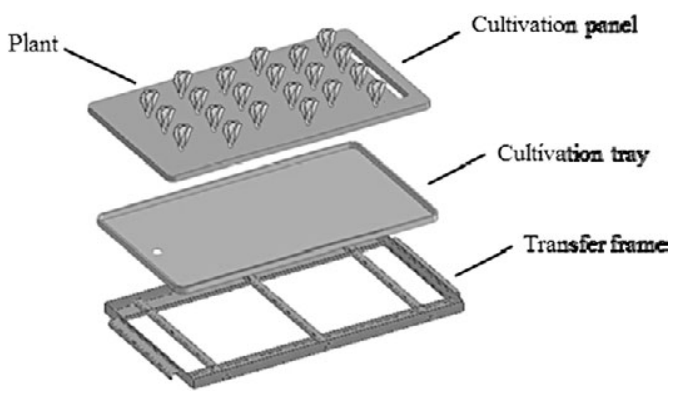

Fig. 3 The plants, cultivation panel, and cultivation tray.

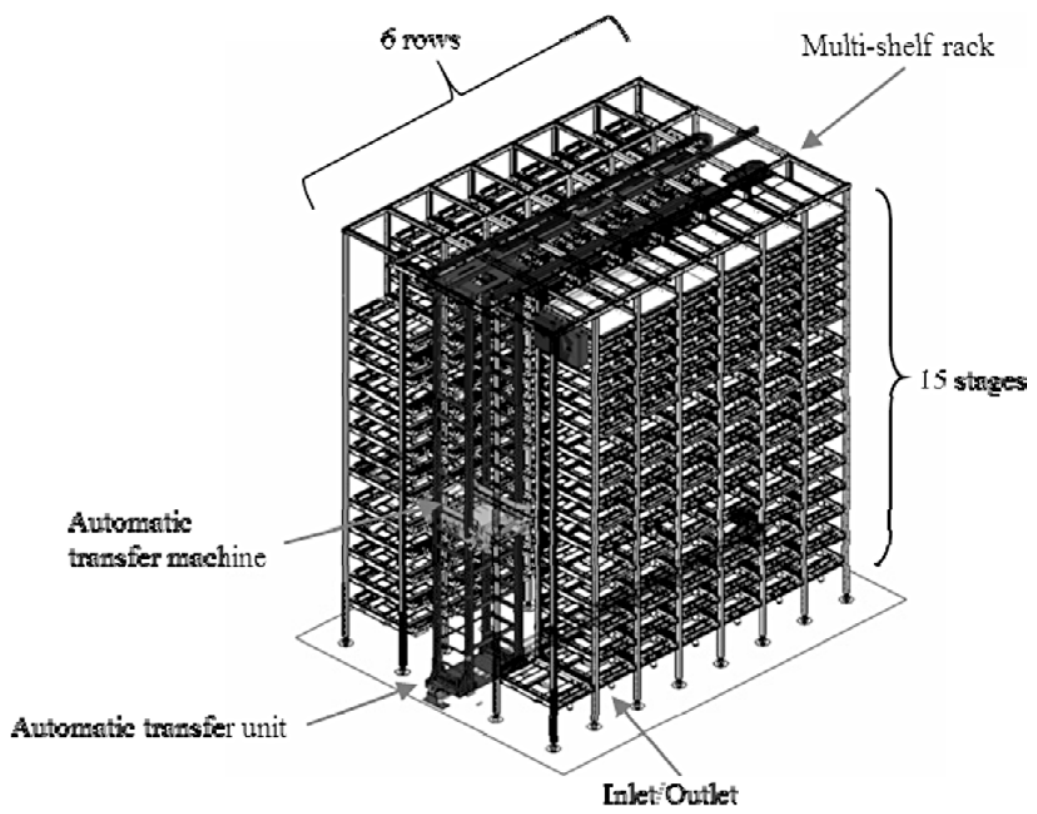

Fig. 2 Multi-story Warehouse Type System. Dimensions: $4300(\mathrm{~W}) \times 6800(\mathrm{D}) \times 6700(\mathrm{H})(\mathrm{mm})$. 


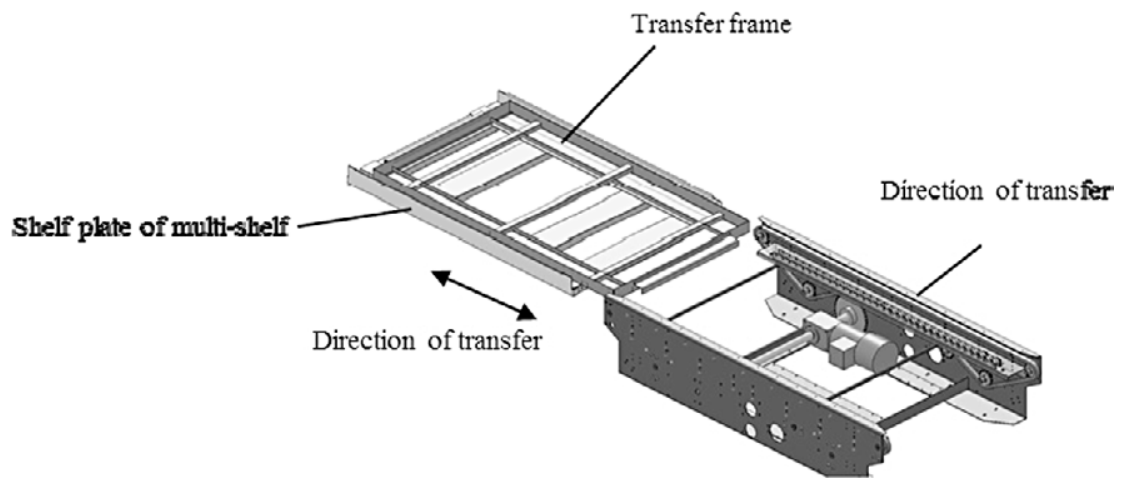

Fig. 4 Transfer by the automatic transfer machine.

are always immersed in a nutrient solution that continuously flows from the top cultivation tray down to the bottom cultivation tray in a rack. Then, at transfer the flow is stopped for a short time so that the cultivation trays can be moved without spilling any of the nutrient solution inside.

Figure 4 shows the transportation of the plants in and out, and to and from the multi-shelf rack by the automatic transfer machine. Since the cultivation tray (Fig. 3) is made of thin resin, the metal transfer frame provides integrity, rigidity and durability.

\section{MECHANICAL COMPONENTS}

As with the established factories for industrial products, plant factories are required to be simple, user-friendly, and reliable. Moreover, this operability should be maintained throughout the plants' growing process. To satisfy these requirements, for the verification of automatic functions, all the cultivation processes were taken into consideration.

Figure 5 shows all of the mechanical components of the system. The main frame of the automatic transfer unit in the center has a lightweight ladder-like structure incorporating the automatic transfer mechanism that can freely ascend and descend. It also moves along the rails on the floor, so enabling plants to be freely moved in any of three perpendicular axes: $\mathrm{x}, \mathrm{y}$ and $\mathrm{z}$.

In summary, the basic functions/parts in the system are as follows:

Transfer: Plant movement to or from a shelf performed by the automatic transfer machine.

Ascend/Descend: The automatic transfer machine

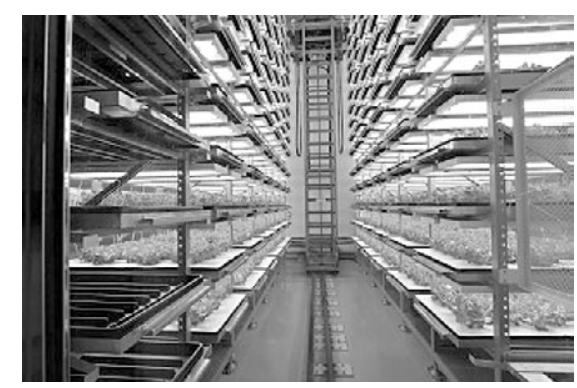

Fig. 5 Automatic transfer unit (center) and multi-shelf rack (both sides). moves in a vertical direction.

Lateral slide: The automatic transfer unit moves laterally.

Water supply and irrigation: This is necessary for hydroponics.

The cultivation shelf that mechanically supports the habitat of the plants.

Each function 1) to 4) above is described in more detail as follows, with particular focus on corrosion protection.

\section{Transfer}

The mechanism developed for insertion and extraction operations is shown in Fig. 6. The insertion operation is when the plants in the multi-shelf rack are loaded onto the automatic transfer machine, and the extraction operation is when the plants in the automatic transfer machine are extracted and loaded into the multi-shelf rack. Figure 6 shows the mechanism details. The pin attached to the chain of the automatic transfer machine engages the L-shaped hook in the transfer frame. During the insertion operation, when the chain moves around in a clockwise direction indicated by the curved arrow, the pin engages the hook, resulting in the transfer frame moving laterally to the right as indicated by the straight arrow. Figure 7 shows an image of the chain-hook mechanism. The chain-hook mechanism used in this study has a simple structure, greatly contributing to the entire system's space-saving specification.

The system mainly depends on hydroponics, in which to assist nutrient absorption through the plant roots, a nutri-

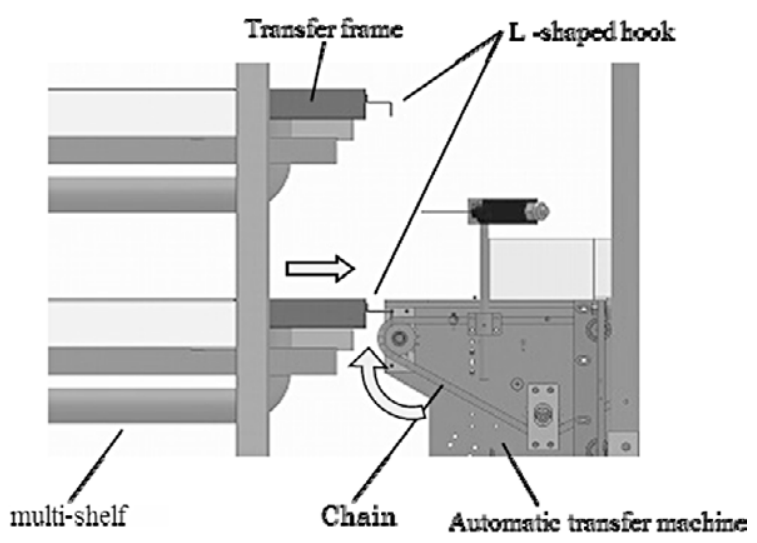

Fig. 6 Transfer mechanism of transfer frame. 


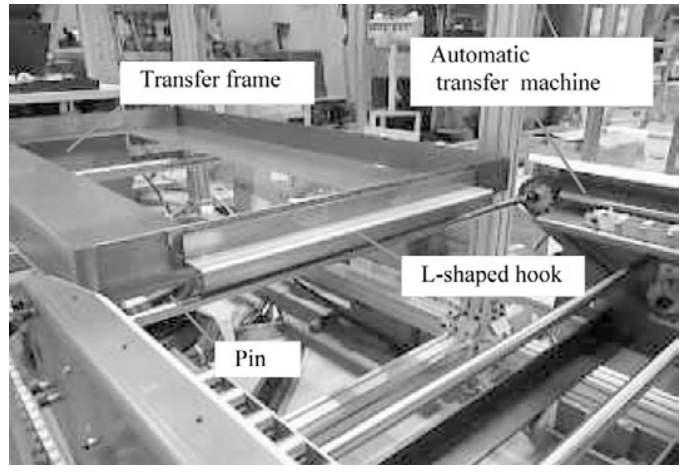

Fig. 7 Chain-hook mechanism.

ent solution is automatically circulated in the cultivation trays. An original transfer method was tried in which the cultivation tray was filled with the nutrient solution, but this could spill easily during transfer, possibly causing the weakly acidic solution to affect the cultivation environment. To solve this problem, and from the electricitysaving aspect to reduce the transfer load, the mechanism has been improved. The solenoid valve for controlling the flow of nutrient solution is closed just before a transfer to stop the flow during transfer. After the transfer is completed the flow is resumed by opening the valve. Since the cultivation tray is transferred without nutrient solution, the plant watering intervals and transfer operations are scheduled in a linked cycle so as not to affect the plants' water requirements for normal growth.

\section{Ascend/Descend}

A notable characteristic of the system is adjustable vertical space between each shelf stage according to the height of the plants. The space is smallest at the uppermost first stage shelf because the plants are still small in height, while the space is large at the lowest final stage shelf for the plants which are ready for harvest.

The ascending and descending movement needs to be halted correctly and precisely at the shelf position of each stage. The engagement of the chain hanging by the laddershaped main frame (Fig. 5) with the rotating sprockets on the automatic transfer machine ensures secure ascending and descending operation.

\section{Lateral slide}

The system horizontally combines twelve rows of shelves, each of which is composed of 15 vertical stages. The same operation is necessary for all of the trays occupying the same stage. This includes the lateral slide operation for transferring trays from/to the inlet/outlet, and in moving between each row of the shelf. During lateral operation, the ladder frame and the automatic transfer machine move along the guide rail.

To facilitate adjustment work during installation, the basic configuration uses a simple mechanism in which the automatic transfer unit moves along using a guide rail. For cost reduction, the design of the ladder-shaped main frame, shown in Fig. 8, has been optimized using finite element method (FEM) analysis for structural simplicity using the minimum quantity of materials under conditions simulating actual use. Figure 8 also shows the degree of frame
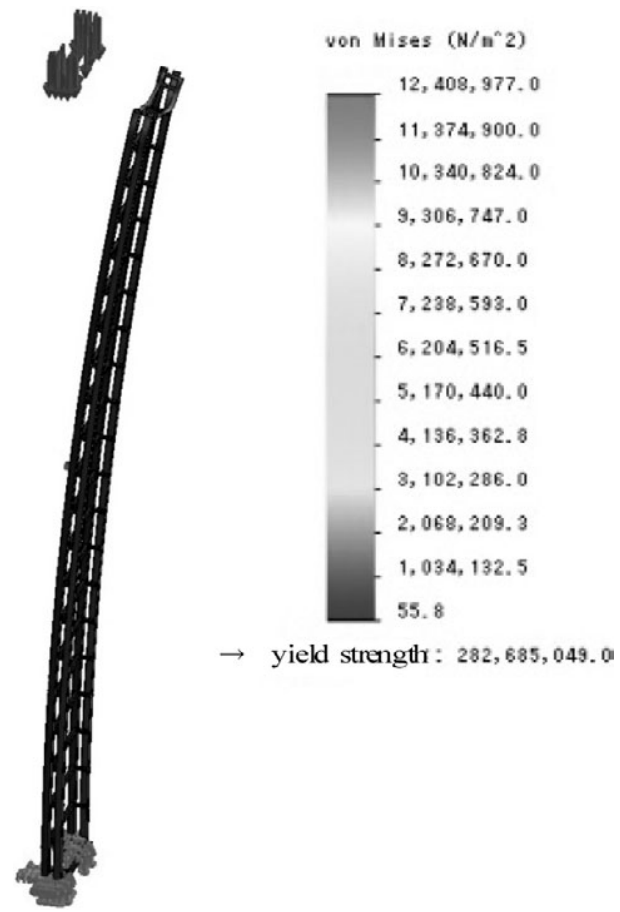

Fig. 8 Example of structural analysis of the ladder frame material using FEM. (The top part is shown deformed for clarity.)

deformation. We have confirmed that this calculation result demonstrates that this structure allows smooth operation of the system during actual use. A guide rail is also installed in the ceiling (upper guide rail) to make the slant of automatic transfer unit adjustable, and the cables necessary for transfer, ascend/descend, and lateral slide are stored in the upper parts of the automatic transfer unit to protect the system from electric current leakage that might possibly arise because of nutrient solution spillage.

In the current system, the lateral sliding drive section is not installed in the ceiling area because of problems of accessibility for maintenance; instead protection measures against falling contaminating substances (such as plant debris, leaves and roots, and spillage of nutrient solution) have been incorporated so that this section can be safely installed on the floor.

Water supply and irrigation (mechanism for circulation of nutrient solution)

When transferring plants, the circulation of nutrient solution is stopped just before the transfer; however, for satisfactory plant growth, the absence of nutrient solution cannot be tolerated for too long. Therefore, the operation sequence of the automatic transfer unit has been set from both the points of view of transfer efficiency (energy/tray transfer) and plant growth rate conditions (plant growth completed per day) by running a number of repeated simulations with the sequences.

More specifically, a timing chart of plant growth factors was drawn up to determine time, count, and interval for the water supply, as well as other transfer conditions which influence plant growth, such as illumination time.

Figure 9 shows an example of simulated operation of 


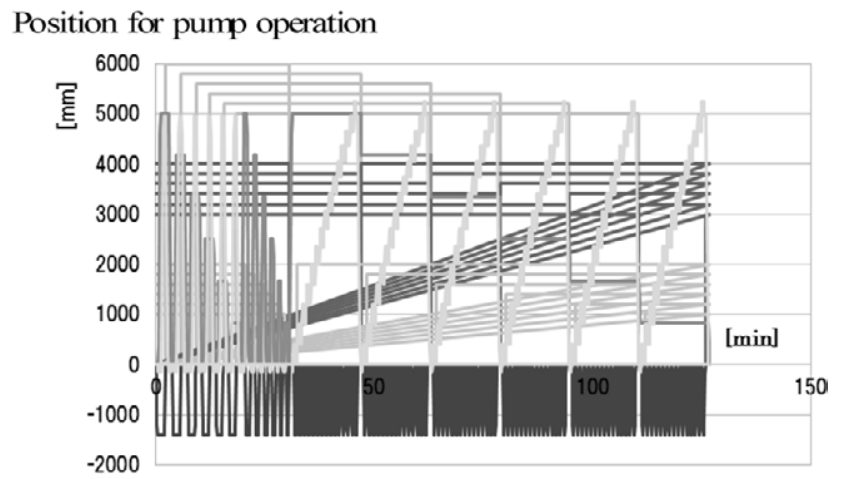

Fig. 9 Example of simulated operation of the timing of the water supply valve opening and the operation of the transfer machine.

the timing of the water supply valve opening and the operation of the transfer machine.

\section{Corrosion protection}

Anti-corrosion measures are necessary for plant factories, to mitigate the high humidity encountered with some cultivation methods or because of leakage, splashing, dripping of the weakly acidic nutrient solutions. For the burgeoning use of these factories cost reduction is a pressing issue; expensive materials such as stainless steel for mechanical design have been replaced by paints with corrosion protection properties. The multi-shelf rack is especially prone to contamination with nutrient solution, and so its steel structure has been hot dip galvanized, providing high corrosion resistance. At the top of the automatic transfer machine, there is an umbrella-shaped cover to protect against drips of nutrient solution, and at the bottom, an anti-drip and anti-splash system to assist the smooth transit of collected solution away from the automatic transfer machine. The motors and the electrical instrumentation are water-proofed to reduce susceptibility to electric current leakage due to moisture ingress.

\section{CONTROL SECTION}

\section{Basic configuration of drive section}

The drive section controller employs a method of driving the induction motor using a general-purpose inverter for lower cost and easier maintenance than a servo motor and/or stepper motor, because the structure of the induction motor is simpler than that of the others. A rotary encoder is connected to the motor shaft, to enable measurement of distance moved, and to allow position control using an inexpensive PLC (Programmable Logic Controller).

\section{Operation monitoring}

The operation status corresponding to the cultivation process stages is updated in real-time on a display screen as shown in Fig. 10. Whether automatic or manual, the operation can be displayed along with other information such as the name of each task in progress and the transfer status. Figure 10 shows a display example during a transfer.

The stop position in each rack is detected by counting the pulse number of the encoder (manufactured by OMRON Co., Ltd., model N6C3-CWZ5GH). The number of pulse depends on the position of each rack. Position control is given by the induction motor to drive the elevating machine comprised of chain and sprocket, controlled by the inverter. The stop position accuracy when driving the transfer machine to keep the nutrition liquid in the cultivation tray is within $\pm 1 \mathrm{~mm}$ at the speed of 140 $\mathrm{mm} \mathrm{s}^{-1}$, which is considered to enable smooth loading/unloading to each rack.

The system operation requires carefully set timing between a transfer and nutrient solution circulation. Figure 11 shows an example of a monitoring screen displaying the supply status of nutrient solution. Figure 11 shows a monitor displaying information about whether cultivation is present or not using green and blue colors and the status of irrigation at the top, middle, and bottom of the multi-shelf rack.

\section{Sensing}

The sensing function described below has been provided in consideration of the characteristics of plant factories and to assure safe operation of the system.

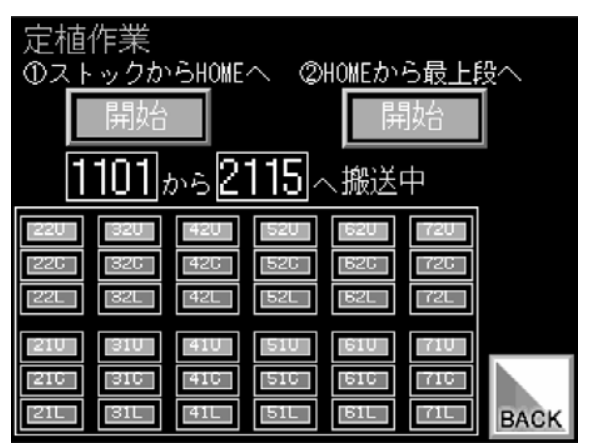

Fig. 10 Display example during transfer.

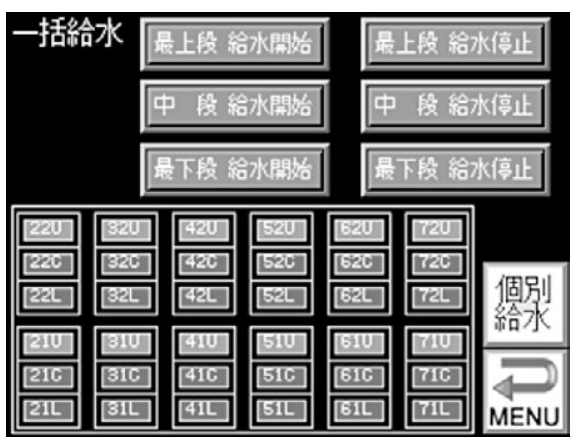

Fig. 11 Example of monitoring screen of water-supply operation. 
1) Presence of cultivation tray on the automatic transfer machine

Two proximity sensors are installed on the automatic transfer machine (Fig. 12, Part A). If both of them are not $\mathrm{ON}$ at the same time, the "Position Error" process will be followed indicating that the cultivation tray is not at the expected position, so the whole system must be halted.

2) Presence of cultivation tray inside the multi-shelf rack

When an attempt is made to insert another cultivation tray into an already occupied shelf space from the automatic transfer machine, or when an attempt is made to transfer a non-existent cultivation tray from a shelf to the automatic transfer machine, a photoelectric sensor on the automatic transfer machine (Fig. 12, Part-B) detects this situation, and starts the "cultivation tray transfer error" process.

3) Presence of a protruding cultivation tray in the multi-shelf rack

A spring wire or sensor (Fig. 12, Part-C) detects whether the cultivation tray protrudes from the multi-shelf rack. When the protrusion exceeds a tolerance limit, the "retraction error" process is performed.

\section{Monitoring}

A notable characteristic of the system is two monitoring cameras installed on the automatic transfer machine to enable inspection and monitoring of plant growth, to look for disease, and to check transfer status. Image information received from the cameras is transmitted with radio communication to notebook PCs or other communication equipment terminals, allowing real-time monitoring. As necessary, camera position (elevation angle or rotation) can be freely adjusted from the above-mentioned terminals.

Previous plant factories mainly depended on monitoring and visual checks to be performed by workers, whereas operations in the multi-shelf rack require work at an elevated location, involving a considerable risk and are therefore not practical. The introduction of monitoring cameras has enabled remote monitoring of plants within the confines of the multi-shelf rack.

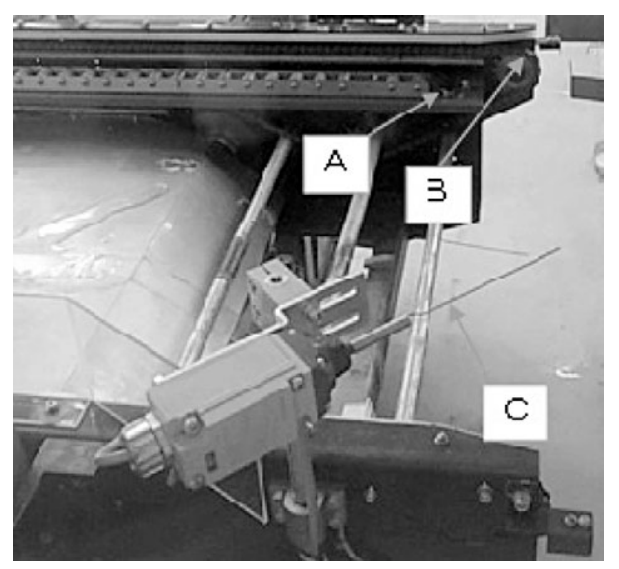

Fig. 12 Sensor installed on the automatic transfer machine.
In this cultivation system, all cultivation trays are moved every day. This is the reason why no special monitoring time is needed and simply by transferring each tray, monitoring of all the cultivation trays in the multi-shelf rack is possible. Figure 13 shows the camera installed on the automatic transfer machine.

\section{ACTUAL OPERATION STATUS}

This system maintains a production rate of 250 lettuces every day for approximately one year, demonstrating

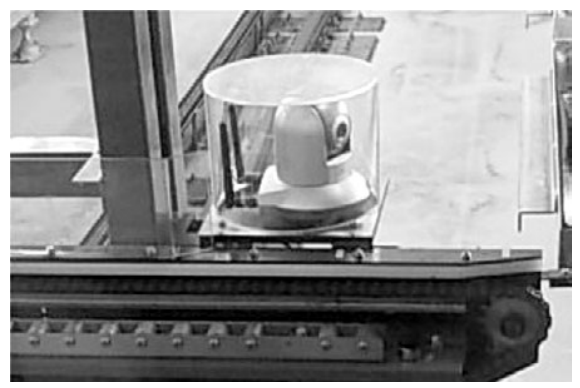

Fig. 13 Monitoring camera installed on the automatic transfer machine.

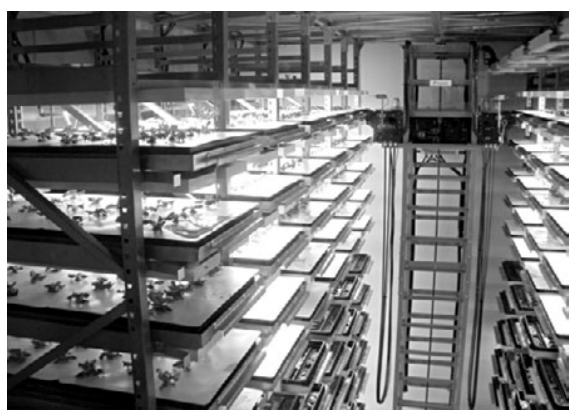

Fig. 14 Transfer to the uppermost level of the multi-shelf rack.

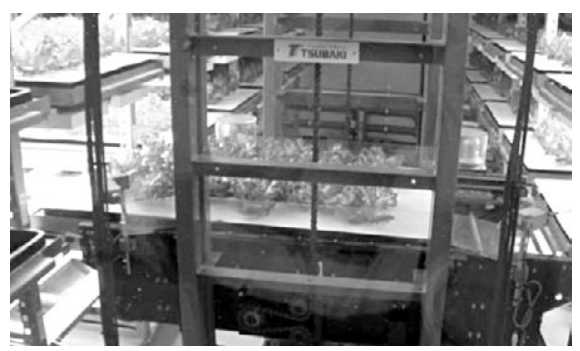

Fig. 15 Transfer to the next stage (one shelf lower) in the multi-shelf rack.

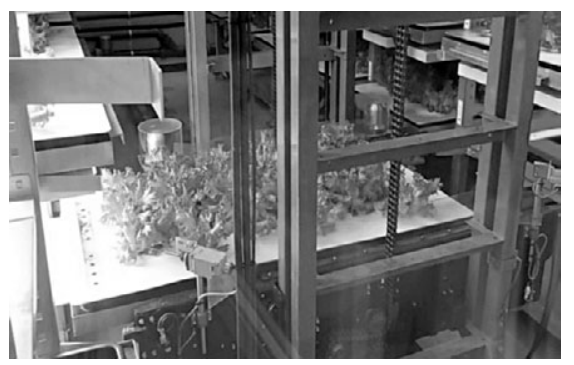

Fig. 16 Transfer to the inlet/outlet from the last stage (lowest shelf) of multi-shelf rack. 


\section{AUTOMATIC PLANT CULTIVATION SYSTEM}

the capacity requirement of a plant factory. Images of actual operation of the system are shown in Figs. 14 to 16.

1) Initial stage of growth (Fig. 14)

This shows a cultivation tray being transferred from the automatic transfer machine to the uppermost level of the multi-shelf rack. Using this cultivation system, the total labor time is reduced by as much as two-thirds.

2) Intermediate stage of growth (Fig. 15)

3) At harvest (Fig. 16)

\section{FUTURE TASKS}

The leaf lettuce cultivation study using the system described herein has proceeded forward for practical application as the academic-industrial cooperation study in Osaka Prefecture University, yielding 250 lettuces per day. The total number of lettuces produced so far has been approximately 200,000.

To obtain a competent automatic cultivation system, we have been pursuing other objectives for improvement, such as a further reduction in production cost and optimization of the cultivation environment including the light source.

The knowledge gained by this study has provided enough for practical application of the technology; our next goal is standardization and/or modularization of the equipment to accommodate a large-sized application, so that we can develop a flexible system design according to production scale requirements in the field.

\section{CONCLUSION}

The multi-shelf rack type cultivation system introduced in this paper is characterized by its substantially increased unit-crop yields when compared to outdoor or glass house cultivation, which are subject to uncontrollable environmental effects that may be less than optimal, such as sunlight exposure and ambient temperatures.

Incorporating automated technology into the system has eliminated workers' risky or troublesome operations, such as simple repetitive tasks in cramped or elevated locations, all of which are generally required in a conventional plant factory. Moreover, any human intervention should require strict consideration for preventing contamination from outside by bacteria hazardous to plants. Without this intervention, bacteria-free, clean cultivation is possible.

Agriculture in Japan has so far been mainly focused on domestic demand, yet the current social conditions arguably point to globalization as the next challenge. Dutchstyle automation technology is already ahead in glass house cultivation. We will further address the development of new-generation cultivation systems which are globally competitive, and responsive to the demands of future customers.

\section{REFERENCES}

Hashimoto, Y. 1985. New processes in cultivation for plants. J. SICE. 24: 947-952.

Ogura, T. 2011. Operation that should be automated and should not be automated in plant factory for leaf vegetables. Sci. High Technol. Agric. 23: 37-43.

Ohara, H. 2012. Automatic plant cultivation system. Proceedings of the Japanese Society of Agricultural Biological and Environmental Engineers and Scientists annual meeting. September, Tokyo, 310-311.

Ohara, H., Hirai, T., Kouno, K. 2012. Tsubaki Tech. Rev. 22: 25-30.

Takatsuji, M., Oguni, K. 1983. Trend and problems in vegetable factory. J. Jpn. Soc. Mechanical Eng. 86: 109-113. 